\title{
Teleparallel Dark Energy with Purely Non-minimal Coupling to Gravity
}

\author{
Je-An Gu, ${ }^{1, *}$ Chung-Chi Lee, $2,+$ and Chao-Qiang Geng $2,3,+$ \\ ${ }^{1}$ Leung Center for Cosmology and Particle Astrophysics, \\ National Taiwan University, Taipei, 10617 Taiwan(R.O.C) \\ ${ }^{2}$ Department of Physics, National Tsing Hua University, Hsinchu, Taiwan 300 \\ ${ }^{3}$ National Center for Theoretical Sciences, Hsinchu, Taiwan 300
}

\begin{abstract}
We propose the simplest model of teleparallel dark energy with purely a non-minimal coupling to gravity but no self-potential, a single model possessing various interesting features: simplicity, selfpotential-free, the guaranteed late-time cosmic acceleration driven by the non-minimal coupling to gravity, tracker behavior of the dark energy equation of state at earlier times, a crossing of the phantom divide at a late time, and the existence of a finite-time future singularity. We find the analytic solutions of the dark-energy scalar field respectively in the radiation, matter, and dark energy dominated eras, thereby revealing the above features. We further illustrate possible cosmic evolution patterns and present the observational constraint of this model obtained by numerical analysis and data fitting.
\end{abstract}

PACS numbers: 95.36.+x, 04.50.Kd, 98.80.Es

\footnotetext{
* jagu@ntu.edu.tw

$\dagger$ g9522545@oz.nthu.edu.tw

‡ geng@phys.nthu.edu.tw
} 


\section{INTRODUCTION}

The accelerating expansion of the present universe discovered in 1998 is one of the most important puzzles yet to be solved [1]. Its solution hopefully will lead us to a new revolution in physics of this century. A positive cosmological constant, as a geometrical and/or an energy component of the universe, gives the simplest explanation that fits the current observational results. In addition, an energy source of anti-gravity dubbed "dark energy" and the modification of gravity provide two intriguing approaches to the solution [2].

A simple realization of dynamical dark energy is given by a scalar field minimally coupled to gravity, where the cosmic acceleration is driven by potential energy [3, 4] or noncanonical kinetic energy [5] of the scalar field. For an alternative explanation from modified gravity one may introduce new degrees of freedom, modify the gravity action, or even change the formalism. A simple extension of general relativity (GR) in the first scenario is to introduce a scalar field non-minimally coupled to gravity, e.g., the scalar-tensor theory [6]. Models with non-minimal derivative couplings have also been proposed [7]. For the second scenario a straightforward modification is to invoke a nonlinear function of the Ricci scalar in the gravity action to replace the linear function in GR, i.e., the $f(R)$ theory [8] that can be regarded as one of the scalar-tensor theories. As to the change of the formalism, an example is teleparallel gravity that is formulated with torsion and a curvatureless connection.

Teleparallel gravity can be equivalent to GR when the gravity action invokes simply a linear function of the torsion scalar. To make an extension of teleparallel gravity as modified gravity, i.e. inequivalent to GR, one may follow the two scenarios raised above. A simple extension can be made by introducing a scalar field non-minimally coupled to teleparallel gravity. This can be regarded as a "scalar-teleparallel theory" of gravity, a modification of teleparallel gravity analogous to the scalar-tensor theory as a modification of GR. It has recently been proposed as an alternative dark energy model [9-12] and dubbed "teleparallel dark energy." For the other scenario a straightforward modification of the gravity action can be made by invoking a nonlinear function of the torsion scalar, i.e., the $f(T)$ theory (for a review, see [13]).

In this paper we focus on teleparallel dark energy. The distinct behavior of dark energy in this model has been investigated in [9, 10] where several different potential terms for the self-interaction of the scalar field are chosen. For lack of a guiding principle of choosing 
the self-potential, in this paper we propose the simplest model with no potential. That is, we investigate the "minimal" model of teleparallel dark energy where the scalar field is canonical, massless and noninteracting but non-minimally coupled to teleparallel gravity. With this simple model we attempt to manifest the effect of the non-minimal coupling on the cosmic acceleration, in contrast to the conventional models that attribute the acceleration to potential energy or noncanonical kinetic energy.

In this model we find the analytic solutions of the scalar field in the radiation, matter, and dark energy dominated eras, respectively. These solutions largely facilitate the theoretical studies on the dark energy behavior and help to reveal various interesting features of this model. In addition to the analytic solutions, we also numerically analyze this model and illustrate possible evolution patterns. As to be shown, the numerical solutions truly manifest the features read from the analytic solutions. We then present the observational constraint of this model obtained by the data fitting with the type Ia supernova (SNIa) [14], baryon acoustic oscillation (BAO) [15, 16], and cosmic microwave background (CMB) [17, 18] observational results.

\section{TELEPARALLEL DARK ENERGY WITHOUT SELF-POTENTIAL}

Teleparallel dark energy is played by a canonical scalar field non-minimally coupled to gravity in the framework of teleparallel gravity, which is formulated with the veirbein field $\mathbf{e}_{A}\left(x^{\mu}\right)$, the metric deduced from veirbein $g_{\mu \nu}(x)=\eta_{A B} e_{\mu}^{A} e_{\nu}^{B}$, the curvatureless Weitzenböck connection $\stackrel{\mathrm{w}}{\Gamma}_{\nu \mu}^{\lambda} \equiv e_{A}^{\lambda} \partial_{\mu} e_{\nu}^{A}$, and the corresponding torsion tensor

$$
T_{\mu \nu}^{\lambda} \equiv \stackrel{\mathrm{w}}{\Gamma}_{\nu \mu}^{\lambda}-\stackrel{\mathrm{w}}{\Gamma}_{\mu \nu}^{\lambda}=e_{A}^{\lambda}\left(\partial_{\mu} e_{\nu}^{A}-\partial_{\nu} e_{\mu}^{A}\right) .
$$

The action of teleparallel dark energy reads

$$
S=\int d^{4} x e\left[\frac{T}{2 \kappa^{2}}+\frac{1}{2}\left(\partial_{\mu} \phi \partial^{\mu} \phi+\xi T \phi^{2}\right)-V(\phi)+\mathcal{L}_{m}\right]
$$

where $e \equiv \operatorname{det}\left(e_{\mu}^{A}\right)=\sqrt{-g}, T$ is the torsion scalar defined by

$$
T \equiv \frac{1}{4} T^{\rho \mu \nu} T_{\rho \mu \nu}+\frac{1}{2} T^{\rho \mu \nu} T_{\nu \mu \rho}-T_{\rho \mu}{ }^{\rho} T_{\nu}^{\nu \mu}
$$

$\phi$ is the scalar field, $V(\phi)$ the self-potential, $\mathcal{L}_{m}$ the matter Lagrangian, and $\xi$ the nonminimal coupling constant. In this paper we investigate the simplest model free of selfpotential, i.e., hereafter $V(\phi)=0$. 
For a flat, homogeneous and isotropic background where the space-time is described by the flat Robertson-Walker metric $d s^{2}=d t^{2}-a^{2}(t) \delta_{i j} d x^{i} d x^{j}$ and the scalar field is simply time-dependent, the scalar field and the gravitational field equations [9] read

$$
\begin{aligned}
& \ddot{\phi}+3 H \dot{\phi}+6 \xi H^{2} \phi=0, \\
& H^{2} \equiv\left(\frac{\dot{a}}{a}\right)^{2}=\frac{\kappa^{2}}{3}\left(\rho_{\phi}+\rho_{\mathrm{m}}+\rho_{\mathrm{r}}\right), \\
& \dot{H}=-\frac{\kappa^{2}}{2}\left(\rho_{\phi}+p_{\phi}+\rho_{\mathrm{m}}+4 \rho_{\mathrm{r}} / 3\right),
\end{aligned}
$$

where the matter energy density $\rho_{\mathrm{m}} \propto a^{-3}$, the radiation energy density $\rho_{\mathrm{r}} \propto a^{-4}$, and the energy density and pressure of the scalar field are given by

$$
\begin{aligned}
\rho_{\phi} & =\frac{1}{2} \dot{\phi}^{2}-3 \xi H^{2} \phi^{2}, \\
p_{\phi} & =\frac{1}{2} \dot{\phi}^{2}+3 \xi H^{2} \phi^{2}+2 \xi \frac{d}{d t}\left(H \phi^{2}\right) .
\end{aligned}
$$

We note that for $\xi<0$, the non-minimal coupling may result in negative pressure, thereby anti-gravity, and meanwhile a positive energy density. In particular, the contribution from the term $3 \xi H^{2} \phi^{2}$ entails the cosmological-constant equation of state $w=-1$ and the other

term $2 \xi d\left(H \phi^{2}\right) / d t$ provides no energy density but negative pressure when $H \phi^{2}$ increases with time. Henceforth we consider the case where $\xi<0$ in order to guarantee the positiveness of the energy density. We set the present scale factor $a_{0}=1$ for convenience and without loss of generality.

\section{ANALYTIC SOLUTIONS}

Here we present the analytic solutions of the scalar field in two cases: (a) the case where $H \propto 1 / t$, including the radiation-dominated (RD) and matter-dominated (MD) eras, and (b) the scalar-field-dominated (SD) era.

(a) $H=\alpha / t$, i.e. $a(t) \propto t^{\alpha}$, with constant $\alpha$ :

$$
\phi(t)=C_{1} t^{l_{1}}+C_{2} t^{l_{2}}
$$

where $C_{1,2}$ are constants and

$$
l_{1,2}=\frac{1}{2}\left[ \pm \sqrt{(3 \alpha-1)^{2}-24 \xi \alpha^{2}}-(3 \alpha-1)\right]
$$


In $\mathrm{RD}, \alpha=1 / 2$; in $\mathrm{MD}, \alpha=2 / 3$. For $\xi<0$, the power-index $l_{1}$ is positive and $l_{2}$ negative, corresponding to an increasing mode and a decreasing mode, respectively.

Hereafter we take into consideration only the increasing mode, i.e., $\phi(t)=C_{1} t^{l_{1}}$. The energy density, pressure, and equation of state corresponding to this solution are given by

$$
\begin{aligned}
\rho_{\phi} & =\left[l_{1}^{2} / 2-3 \xi \alpha^{2}\right] C_{1}^{2} t^{2\left(l_{1}-1\right)}, \\
p_{\phi} & =\left[l_{1}^{2} / 2+3 \xi \alpha^{2}+2 \xi \alpha\left(2 l_{1}-1\right)\right] C_{1}^{2} t^{2\left(l_{1}-1\right)}, \\
w_{\phi} & \equiv \frac{p_{\phi}}{\rho_{\phi}}=1+\frac{4 \xi \alpha}{l_{1}}=-1+\frac{2\left(1-l_{1}\right)}{3 \alpha} .
\end{aligned}
$$

Consequently, one obtains

$$
\begin{aligned}
& \mathrm{RD}: w_{\phi}=\frac{1}{3}(2-\sqrt{1-24 \xi}), \quad \rho_{\phi} \propto a^{-5+\sqrt{1-24 \xi}} \\
& \mathrm{MD}: w_{\phi}=\frac{1}{2}(1-\sqrt{1-32 \xi / 3}), \quad \rho_{\phi} \propto a^{(-9+\sqrt{9-96 \xi}) / 2} .
\end{aligned}
$$

To sketch the behavior of teleparallel dark energy in RD and MD, in Table 1 we consider four different $\xi$ as the examples for demonstration. The $\xi$ values under consideration are within the range $[-1,0)$ because the case where $\xi<-1$ is rather disfavored by observational results about the cosmic expansion. The four values of $\xi$ are so chosen that in $\mathrm{RD}$ or MD the dark energy density $\rho_{\phi}$ behaves like the familiar energy sources such as radiation, matter, or a cosmological constant: (i) When $\xi=0^{-}$, teleparallel dark energy tracks the dominant energy components respectively in RD and MD, a behavior similar to the tracker quintessence [19] (ii) When $\xi=-1 / 8$, in $\mathrm{RD} \rho_{\phi}$ behaves like matter and in MD it drops slower than matter. (iii) When $\xi=-3 / 4$, in $\mathrm{RD} \rho_{\phi}$ decreases rather slowly and in MD it is constant. (iv) When $\xi=-1$, in $\mathrm{RD} \rho_{\phi}$ is constant and in MD it increases as phantom.

TABLE I. Explicit solutions of the scalar field in RD and MD with different values of $\xi$.

\begin{tabular}{|c||c|c||c|c||c|c||c|c|}
\hline$\xi$ & \multicolumn{2}{|c||}{$(\mathrm{i}) 0^{-}$} & \multicolumn{2}{c||}{ (ii) $-1 / 8$} & \multicolumn{2}{c||}{ (iii) $-3 / 4$} & \multicolumn{2}{c|}{ (iv) -1} \\
\hline \hline Era & $\mathrm{RD}$ & $\mathrm{MD}$ & $\mathrm{RD}$ & $\mathrm{MD}$ & $\mathrm{RD}$ & $\mathrm{MD}$ & $\mathrm{RD}$ & $\mathrm{MD}$ \\
\hline$l_{1}$ & $-3 \xi$ & $-8 \xi / 3$ & $+1 / 4$ & +0.264 & 0.840 & +1 & +1 & +1.208 \\
\hline$w_{\phi}$ & $1 / 3+4 \xi$ & $-8 \xi / 3$ & 0 & -0.264 & -0.786 & -1 & -1 & -1.208 \\
\hline$\rho_{\phi} \propto$ & $a^{-4} a^{-12 \xi}$ & $a^{-3} a^{-8 \xi}$ & $a^{-3}$ & $a^{-2.21}$ & $a^{-0.641}$ & Constant & Constant & $a^{0.623}$ \\
\hline
\end{tabular}

The analytic solution, as well as the examples in Table \, shows several interesting features: The dark energy equation of state $w_{\phi}$ is a constant simply determined by the sole 
model parameter $\xi$ but insensitive to the initial condition of the scalar field, i.e., a tracker behavior of the dark energy equation of state 1 For $\xi<0, w_{\phi}$ is smaller than that of the dominant energy component, $w_{\alpha}=-1+2 /(3 \alpha)$, at all times in RD and MD. Accordingly, the dark energy density $\rho_{\phi}$ decreases slower than the dominant energy density $\rho_{\alpha}$ and therefore the late-time domination of dark energy (SD) is guaranteed. For larger $|\xi|, w_{\phi}$ is smaller and $\rho_{\phi} / \rho_{\alpha}$ increases faster, and therefore SD comes sooner for a fixed initial condition.

The analytic solutions in RD and MD describe the universe at earlier times. At later times, the universe is dominated by both matter and dark energy (MSD), e.g., in the present epoch, and then dominated solely by dark energy (SD) in the future. In the following we attempt to analytically integrate the field equations in both of the later eras (MSD and SD).

\section{(b) Scalar field domination:}

For the universe dominated by matter and dark energy, we deduce an integrable equation from Eq. (4):

$$
\begin{aligned}
\frac{d}{d t}\left[F(\phi) a^{3} H\right] & =\frac{\kappa^{2}}{2} \rho_{\mathrm{m}} a^{3}=\frac{3}{2} H_{0}^{2} \Omega_{\mathrm{m} 0}, \\
F(\phi) & \equiv 1+\kappa^{2} \xi \phi^{2},
\end{aligned}
$$

where $H_{0}$ and $\Omega_{\mathrm{m} 0}$ are the Hubble constant and the matter density fraction at the present time, respectively. Its solution gives a relation:

$$
F(\phi) a^{3} H=\frac{3}{2} H_{0}^{2} \Omega_{\mathrm{m} 0} t+C_{3},
$$

where $C_{3}$ is an integration constant. This can hardly be further integrated analytically.

For the universe dominated by dark energy solely, Eq. (12) gives a simpler relation with $\Omega_{\mathrm{m} 0}=0$ :

$$
F(\phi) a^{3} H=C_{3}
$$

From this relation and the field equations we obtain the second integrable equation,

$$
\frac{1}{F(\phi)}\left(\frac{d \phi}{d \ln a}\right)^{2}=\frac{6}{\kappa^{2}},
$$

from which one can straightforwardly obtain the solution,

$$
\begin{aligned}
& \phi(a)= \pm \frac{\sin \theta}{\sqrt{-\kappa^{2} \xi}}, \\
& \theta(a) \equiv \sqrt{-6 \xi} \ln a+C_{4},
\end{aligned}
$$

1 This is different from tracker quintessence [19] where it is the scalar field (thereby the dark energy density and its equation of state) that possesses the tracker behavior. 
and then the equation of state,

$$
w_{\phi}=-1-\sqrt{-32 \xi / 3} \tan \theta
$$

where $C_{4}$ is an integration constant.

This solution predicts a future singularity where both the expansion rate $H$ and the dark energy equation of state $w_{\phi}$ go to infinity. It is one kind of finite-time future singularities, i.e. "type III" singularity classified in Ref. [20]. The singularity occurs when $\theta=(n+1 / 2) \pi$ for integer $n$, i.e., $\phi= \pm 1 / \sqrt{-\kappa^{2} \xi}$ and $F(\phi)=0$. Between two singularities, e.g., for $-\pi / 2<\theta<\pi / 2, \phi$ is monotonic and $w_{\phi}$ monotonically decreases from $+\infty$ to $-\infty$ along with the expansion, experiencing the crossing of the phantom divide $w_{\phi}=-1$ at $\theta=0$.

\section{POSSIBLE EVOLUTION PATTERNS AND DATA FITTING}

Here we illustrate the evolution patterns of the dark energy equation of state $w_{\phi}$ for a wide range of initial conditions by numerically solving the field equations (4). These numerical solutions will manifest the interesting features we have read from the analytic solutions, particularly the feature that $w_{\phi}$ approaches the constant tracker values in RD and MD, leaves the tracker and starts decreasing when the universe is leaving MD for SD, keeps decreasing and crosses the phantom divide in SD, and goes to negative infinity at a finite time eventually. In addition, we confront this self-potential-free teleparallel dark energy model with the observational data that are relevant to the cosmic expansion, including SNIa [14], BAO [15, 16] and CMB [17, 18], thereby obtaining the constraint on the sole model parameter, i.e. the non-minimal coupling constant $\xi$, as well as the cosmological parameters.

For illustrating the evolution patterns of $w_{\phi}$ numerically, we consider the case where $\xi=-0.35$ and show $w_{\phi}(\log (a))$ in Fig. 1 for different initial conditions. For the same case we also show the present values of $\left(\Omega_{m}, w_{\phi}\right)$ in Fig. 2. We consider two sets of initial conditions at the initial time $\log (a) \simeq-8.69$ (i.e. $\ln (a)=-20$ ): (i) $\kappa \phi=0$ and $\kappa \phi^{\prime} \equiv d(\kappa \phi) / d \ln a \in$ $\left[10^{-50}, 2.5 \times 10^{-8}\right]$ (solid line), and (ii) $\kappa \phi^{\prime}=0$ and $\kappa \phi \in\left[10^{-50}, 1.2 \times 10^{-8}\right]$ (dashed line). For these two kinds of initial conditions, initially $w_{\phi}$ is unity and $1 / 3$, respectively. Then, as shown in Fig. 1, it quickly evolves to the RD tracker value, -0.36 , along with the domination of the increasing mode of $\phi(t)$-field over the decreasing mode. It reaches the tracker value around $\log (a)=-8$. When the universe gradually leaves RD and enters MD, $w_{\phi}$ gradually 
decreases to the MD tracker value, -0.58 . Later, when teleparallel dark energy starts to dominate, $w_{\phi}$ leaves the MD tracker and decreases monotonically. Finally, $w_{\phi}$ crosses the phantom divide $\left(w_{\phi}=-1\right)$ and goes to the singularity as expected.

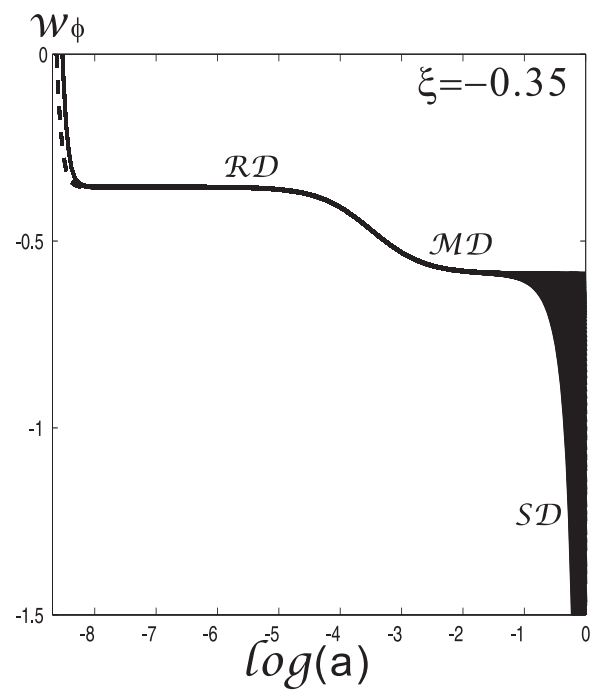

FIG. 1. Evolution of the dark energy equation of state, $w_{\phi}(\log (a))$, with $\xi=-0.35$. The black area in SD is formed by the trajectories of $w_{\phi}(\log (a))$ w.r.t. a wide range of initial conditions.

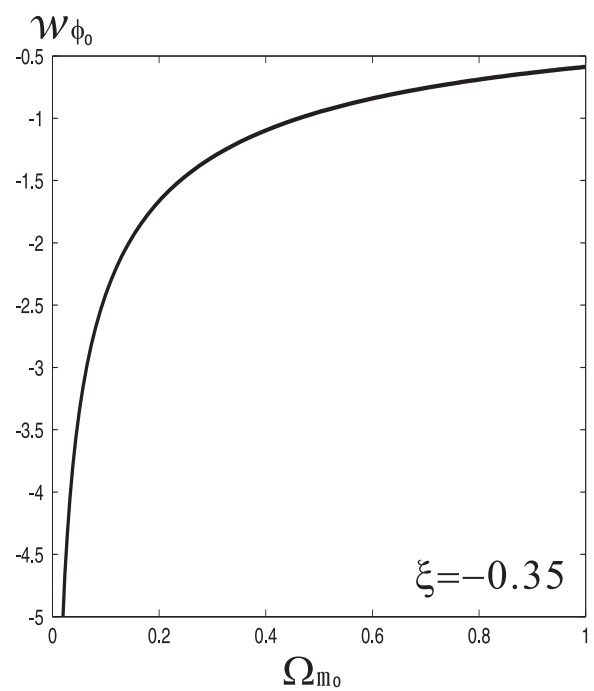

FIG. 2. The present values of $\left(\Omega_{m}, w_{\phi}\right)$ for a wide range of initial conditions with $\xi=-0.35$. The curve shows a relation between these two quantities, as a consequence of the tracker behavior of $w_{\phi}$ for a flat universe with given $\xi$.

Because of the tracker behavior of $w_{\phi}$, the evolution of dark energy is simply determined 
by two parameters, the model parameter $\xi$ and the integration constant $C_{1}$ appearing in Eq. (6), where $C_{1}$ may be regarded as the only important initial-condition parameter. Accordingly, for a flat universe with given $\xi$, the present value of $w_{\phi}$ and the present matter density fraction $\Omega_{m 0}$ are related by a single parameter $C_{1}$. This relation is shown in Fig. 2 , where both $\Omega_{m 0}$ and $w_{\phi 0}$ change with the initial condition, i.e. the value of $C_{1}$. The present value of $w_{\phi}$ has an upper bound that depends on $\xi$ and is set by the tracker value of $w_{\phi}$ in MD. Note that the two different sets of initial conditions give the same relation curve.

Following the same procedure of data fitting in Ref. [10], we obtain the observational constraint of our model. Figure 3 shows the $1 \sigma-3 \sigma$ confidence regions in $\left(\Omega_{m 0}, \xi\right)$ (left panel) and $\left(\Omega_{m 0}, w_{\phi 0}\right)$ (right panel) obtained from the SNIa (blue), BAO (green), CMB (red), and the combined (black) data. The best fit to the combined data locates at $\xi \simeq-0.35$ and $\Omega_{\mathrm{m} 0} \simeq 0.28$. The three data sets do not give a good concordance region, indicating the imperfection in simultaneously fitting these data sets. This is possibly due to the feature of this model that $\rho_{\phi}$ may not be negligible at early times and accordingly may significantly affect the $\mathrm{CMB}$ result. We also remark that in contrast the data can be fitted well in the teleparallel dark energy models with potentials in Refs. [9, 10]. This is expected because the models with potentials are more flexible than our simple potential-free model when fitting data.
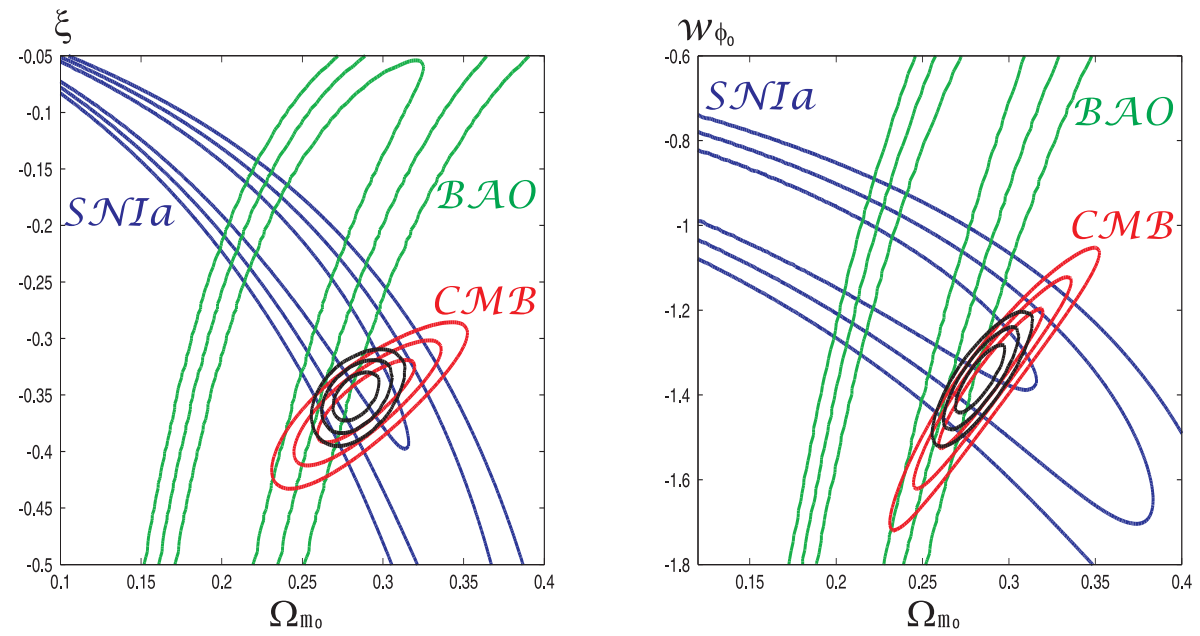

FIG. 3. The $1 \sigma-3 \sigma$ confidence regions in $\left(\Omega_{m 0}, \xi\right)$ (left panel) and $\left(\Omega_{m 0}, w_{\phi 0}\right)$ (right panel) obtained from the SNIa (blue), BAO (green), CMB (red), and the combined (black) data. 


\section{SUMMARY}

We propose the simplest model of teleparallel dark energy where dark energy is played by a scalar field that is canonical, massless and noninteracting but purely non-minimally coupled to teleparallel gravity. We have found the analytic solutions of the scalar field, thereby obtaining the analytic behaviors of the dark energy equation of state, in the radiation, matter, and dark energy dominated eras, respectively. With the analytic solutions we have shown various interesting features of this simple model:

- The cosmic acceleration is driven by the non-minimal coupling to gravity, but not by potential energy or noncanonical kinetic energy as invoked in the conventional scalar-field dark energy models.

- The domination of dark energy, the crossing of the phantom divide, and therefore the occurrence of the cosmic acceleration at late times are destined.

- In the radiation and matter dominated eras the dark energy equation of state is roughly a constant and has tracker behavior, i.e. being insensitive to the initial condition.

- In the dark energy dominated era the dark energy equation of state decreases monotonically and crosses the phantom divide at a late time; its value strongly depends on the initial condition of the scalar field.

- The universe will meet a future singularity with the expansion rate going to infinity within a finite time.

- This model has simply one free parameter, the non-minimal coupling constant $\xi$, for a spatially flat FLRW universe with the present matter density $\Omega_{\mathrm{m} 0}$ fixed.

We have fitted the model parameter $\xi$ with the observational data of SNIa, BAO and CMB in cosmology. The concordance region for all these three data sets is only at the $3 \sigma$ level. It may indicate a slight incapability for this model to describe the early universe. This requires further examination. For further investigations, in addition to cosmological observations, the local gravity tests are expected to give significant constraints on teleparallel dark energy and therefore should be worth detailed studies. 


\section{ACKNOWLEDGMENTS}

The work was supported in part by National Center of Theoretical Science and by National Science Council of R.O.C. under Grants Nos. NSC98-2112-M-002-007-MY3 (JAG) and NSC-98-2112-M-007-008-MY3 (CCL and CQG).

[1] A. G. Riess et al. [Supernova Search Team Collaboration], Astron. J. 116, 1009 (1998). S. Perlmutter et al. [Supernova Cosmology Project Collaboration], Astrophys. J. 517, 565 (1999).

[2] E. J. Copeland, M. Sami and S. Tsujikawa, Int. J. Mod. Phys. D 15, 1753 (2006); M. Li, X. -D. Li, S. Wang and Y. Wang, Commun. Theor. Phys. 56, 525 (2011).

[3] B. Ratra and P. J. E. Peebles, Phys. Rev. D 37, 3406 (1988); R. R. Caldwell, R. Dave and P. J. Steinhardt, Phys. Rev. Lett. 80, 1582 (1998); I. Zlatev, L.-M. Wang and P. J. Steinhardt, Phys. Rev. Lett. 82, 896 (1999).

[4] R. R. Caldwell, Phys. Lett. B 545, 23 (2002).

[5] C. Armendariz-Picon, V. F. Mukhanov and P. J. Steinhardt, Phys. Rev. Lett. 85, 4438 (2000); T. Chiba, T. Okabe and M. Yamaguchi, Phys. Rev. D 62, 023511 (2000).

[6] S. Nojiri and S. D. Odintsov, Phys. Lett. B 599, 137 (2004); G. Allemandi, A. Borowiec, M. Francaviglia and S. D. Odintsov, Phys. Rev. D 72, 063505 (2005).

[7] L. Amendola, Phys. Lett. B 301, 175 (1993); S. Capozziello and G. Lambiase, Gen. Rel. Grav. 31, 1005 (1999); S. Capozziello, G. Lambiase and H. J. Schmidt, Annalen Phys. 9, 39 (2000); S. F. Daniel and R. R. Caldwell, Class. Quant. Grav. 24, 5573 (2007); S. V. Sushkov, Phys. Rev. D 80, 103505 (2009); E. N. Saridakis and S. V. Sushkov, Phys. Rev. D 81, 083510 (2010); C. Gao, JCAP 1006, 023 (2010); L. N. Granda, JCAP 1007, 006 (2010); G. Gubitosi and E. V. Linder, Phys. Lett. B 703, 113 (2011).

[8] S. Nojiri and S. D. Odintsov, eConf C 0602061, 06 (2006) [Int. J. Geom. Meth. Mod. Phys. 4, 115 (2007)]; T. P. Sotiriou and V. Faraoni, Rev. Mod. Phys. 82, 451 (2010); A. De Felice and S. Tsujikawa, Living Rev. Rel. 13, 3 (2010); S. Nojiri and S. D. Odintsov, Phys. Rept. 505, 59 (2011).

[9] C. Q. Geng, C. C. Lee, E. N. Saridakis and Y. P. Wu, Phys. Lett. B 704, 384 (2011).

[10] C. Q. Geng, C. C. Lee and E. N. Saridakis, JCAP 1201, 002 (2012). 
[11] H. Wei, Phys. Lett. B 712, 430 (2012).

[12] C. Xu, E. N. Saridakis and G. Leon, JCAP 1207, 005 (2012).

[13] K. Bamba, S. Capozziello, S. Nojiri and S. D. Odintsov, Astrophys. Space Sci. 342, 155 (2012).

[14] L. Perivolaropoulos, Phys. Rev. D 71, 063503 (2005).

[15] D. J. Eisenstein and W. Hu, Astrophys. J. 496, 605 (1998).

[16] D. J. Eisenstein et al. [SDSS Collaboration], Astrophys. J. 633, 560 (2005).

[17] J. R. Bond, G. Efstathiou and M. Tegmark, Mon. Not. Roy. Astron. Soc. 291, L33 (1997).

[18] W. Hu and N. Sugiyama, Astrophys. J. 471, 542 (1996).

[19] I. Zlatev, L. Wang and P. J. Steinhardt, Phys. Rev. Lett. 82, 896 (1999); P. J. Steinhardt, L. Wang and I. Zlatev, Phys. Rev. D 59, 123504 (1999).

[20] S. Nojiri, S. D. Odintsov and S. Tsujikawa, Phys. Rev. D 71, 063004 (2005). 